\title{
On the Exponentiated Generalized Modified Weibull Distribution
}

\author{
Gokarna Aryal ${ }^{1, a}$, Ibrahim Elbatal $\left.\right|^{b}$ \\ ${ }^{a}$ Department of Mathematics, Computer Science, and Statistics, \\ Purdue University Calumet, USA; \\ ${ }^{b}$ Institute of Statistical Studies and Research, Department of Mathematical Statistics, \\ Cairo University, Egypt
}

\begin{abstract}
In this paper, we study a generalization of the modified Weibull distribution. The generalization follows the recent work of Cordeiro et al. (2013) and is based on a class of exponentiated generalized distributions that can be interpreted as a double construction of Lehmann. We introduce a class of exponentiated generalized modified Weibull (EGMW) distribution and provide a list of some well-known distributions embedded within the proposed distribution. We derive some mathematical properties of this class that include ordinary moments, generating function and order statistics. We propose a maximum likelihood method to estimate model parameters and provide simulation results to assess the model performance. Real data is used to illustrate the usefulness of the proposed distribution for modeling reliability data.
\end{abstract}

Keywords: modified Weibull distribution, order statistics, parameter estimation, reliability, simulation

\section{Introduction}

Statistical distributions are useful to describe and predict real world phenomena. Statistics literature contains hundreds of continuous univariate distributions and example of their successful applications. Recent developments focus on constructing a new class of distribution by inducting one (or more) additional shape parameter (s) to the baseline (standard) distribution which makes the distribution more flexible, especially for studying tail behavior. In the context of life time distributions, if $G(x)$ is the cumulative distribution function (cdf) of the distribution, then the three well known methods of generalizations include:

- Exponentiated-G distribution

- Beta-G distribution

- Kumaraswamy-G distribution

For a given cdf $G(x)$, the exponentiated class of distribution is given by

$$
F_{E}(x)=[G(x)]^{\beta}, \quad \beta>0 .
$$

\footnotetext{
${ }^{1}$ Corresponding author: Department of Mathematics, Computer Science, and Statistics, Purdue University Calumet, USA.

E-mail: aryalg@purduecal.edu

Published 31 July 2015 / journal homepage: http://csam.or.kr

(c) 2015 The Korean Statistical Society, and Korean International Statistical Society. All rights reserved.
} 
Mudholkar and Srivastava (1993) first proposed the exponentiated Weibull distribution to analyze bathtub failure data. Gupta et al. (1998) also proposed a generalization of standard exponential distribution, called exponentiated exponential (EE) distribution. For a full discussion and some of its mathematical properties, see Gupta and Kundu (2001). In a similar manner, Nadarajah and Kotz (2006) also proposed and studied exponentiated gamma, exponentiated Fréchet and exponentiated Gumbel distributions; however, with a slightly different defined cdf of the last two distributions.

The beta distribution generalizes any parent cumulative distribution function $G(x)$ as

$$
F_{B G}(x)=I_{G(x)}(a, b)=\frac{B_{G(x)}(a, b)}{B(a, b)},
$$

where $B_{G(x)}(a, b)=\int_{0}^{G(x)} w^{a-1}(1-w)^{b-1} d w$ is incomplete beta function. The generalization given in (1.2) involves some complexity due to the presence of an incomplete beta function but numerous researchers used this method to generalize some well-known distributions. Some notable references include Nadarajah and Kotz (2004, 2005), Nadarajah and Gupta (2004), Famoye et al. (2005), Eugene et al. (2002), and Hanook et al. (2013).

Generalization using Kumaraswamy distribution also has been discussed by several authors. In this method if $G(x)$ denotes the baseline cdf of a random variable, then a generalized class of distributions can be defined by

$$
F_{K G}(x)=1-\left[1-G(x)^{a}\right]^{b},
$$

where $a>0$ and $b>0$ are two additional shape parameters. Jones (2009) explored the background and genesis of the Kumaraswamy distribution to examine some of the similarities and differences between the beta and Kumaraswamy distributions. The author highlighted several advantages of the Kumaraswamy distribution over the beta distribution. Refer to Jones (2009) for a detailed survey of the Kumaraswamy distribution.

Cordeiro et al. (2013) proposed a new class of exponentiated generalized (EG) distribution that extends the exponentiated type distributions. Given a continuous cdf $G(x)$, the EG class of distributions are given by

$$
F_{E G}(x)=\left[1-\{1-G(x)\}^{\alpha}\right]^{\beta},
$$

where $\alpha>0$ and $\beta>0$ are two additional shape parameters. We note that there is no complicated function in (1.4) in contrast to the beta generalized family. Although this generalization also includes two extra parameters as in the beta generalization but (1.4) is easily tractable.

The baseline distribution $G(x)$ is a special case of (1.4) when $\alpha=\beta=1$. Similarly, it is important to note that for $\alpha=1$ we have Lehmann type $I$ distribution and for $\beta=1$ we have Lehmann type II distribution. Thus (1.4) encompasses both Lehmann type $I$ and Lehmann type $I I$ distributions (Lehmann, 1953).

The probability density function (pdf) of the new class can be obtained by differentiating (1.4) with respect to $x$ and takes the form

$$
f(x, \alpha, \beta)=\alpha \beta g(x)[1-G(x)]^{\alpha-1}\left[1-\{1-G(x)\}^{\alpha}\right]^{\beta-1} .
$$

The EG family of densities (1.5) allows for greater flexibility of its tails and can be widely applied to many areas of engineering and biology. 
As described in Cordeiro et al. (2013) the class of EG distributions shares an attractive physical interpretation whenever $\alpha$ and $\beta$ are positive integers. Consider a device made of independent components in a parallel system with each component made of independent subcomponents identically distributed according to $G(x)$ in a series system. The device fails if all components fail and each component fails if any subcomponent fails. Let $X_{j 1}, \ldots, X_{j \alpha}$ denote the lifetimes of the subcomponents within the $j^{\text {th }}$ component, $j=1, \ldots, \beta$, with common cdf $G(x)$. Let $X_{j}$ denote the lifetime of the $j^{\text {th }}$ component and let $X$ denote the lifetime of the device. Therefore, the cdf of $X$ is

$$
\begin{aligned}
P(X \leq x) & =P\left(X_{1} \leq x, \ldots, X_{\beta} \leq x\right)=P\left(X_{1} \leq x\right)^{\beta} \\
& =\left[1-P\left(X_{1}>x\right)\right]^{\beta}=\left[1-P\left(X_{11}>x, \ldots, X_{1 \alpha}>x\right]^{\beta}\right. \\
& =\left[1-P\left(X_{11}>x\right)^{\alpha}\right]^{\beta}=\left[1-\left\{1-P\left(X_{11} \leq x\right)\right\}^{\alpha}\right]^{\beta} .
\end{aligned}
$$

and the lifetime of the device obeys the EG family of distributions. In this paper, we proposed a new distribution based on the EG family- called an exponentiated generalized modified Weibull (EGMW) distribution.

Modified Weibull (MW) distribution is one of the most important distributions in lifetime modeling, and some well-known distributions are special cases of it. This distribution was introduced by Lai et al. (2003) to which we refer the reader for a detailed discussion as well as applications of the MW distribution (in particular, the use of a real data set representing failure times to illustrate the modeling and estimation procedure). Sarhan and Zaindin (2009) also introduced the MW distribution that be used to describe several reliability models. It has three parameters, two scale and one shape parameters. Carrasco et al. (2008) recently extended MW distribution by adding another shape parameter and introducing a four parameter generalized MW (GMW) distribution.

A random variable $X$ has have MW distribution if its cumulative distribution function (cdf) is given by

$$
G(x)=1-e^{-\theta x-\mu x^{\lambda}}, \quad x \geq 0,
$$

where $\lambda>0, \theta, \mu \geq 0$, such that $\theta+\mu>0$. Here $\theta$ is a scale parameter, while $\mu$ and $\lambda$ are shape parameters. The corresponding probability density function (pdf) is

$$
g(x)=\left(\theta+\mu \lambda x^{\lambda-1}\right) e^{-\theta x-\mu x^{\lambda}},
$$

and the hazard function is given by

$$
\begin{aligned}
h(x) & =\frac{g(x)}{1-G(x)}=\frac{\left(\theta+\mu \lambda x^{\lambda-1}\right) e^{-\theta x-\mu x^{\lambda}}}{e^{-\theta x-\mu x^{\lambda}}} \\
& =\left(\theta+\mu \lambda x^{\lambda-1}\right) .
\end{aligned}
$$

One can verify from (1.8) that: (i) the hazard function is constant when $\lambda=1$; (ii) the hazard rate function is decreasing when $\lambda<1$; and (iii) the hazard function will be increasing if $\lambda>1$.

The rest of the paper is organized as follows. In Section 2, we define the EGMW distribution, discuss some of its sub-models and provide their cumulative distribution function (cdf). Section 3 discusses structural and mathematical properties of EGMW distribution such as the quantile and moments. The distribution of the order statistics is studied in Section 4. Parameter estimation procedures using method of maximum likelihood estimates are presented in Section 5. Section 6 discusses simulation results to assess the performance of the proposed maximum likelihood estimation procedure. The application to model reliability data are discussed in Section 7. 


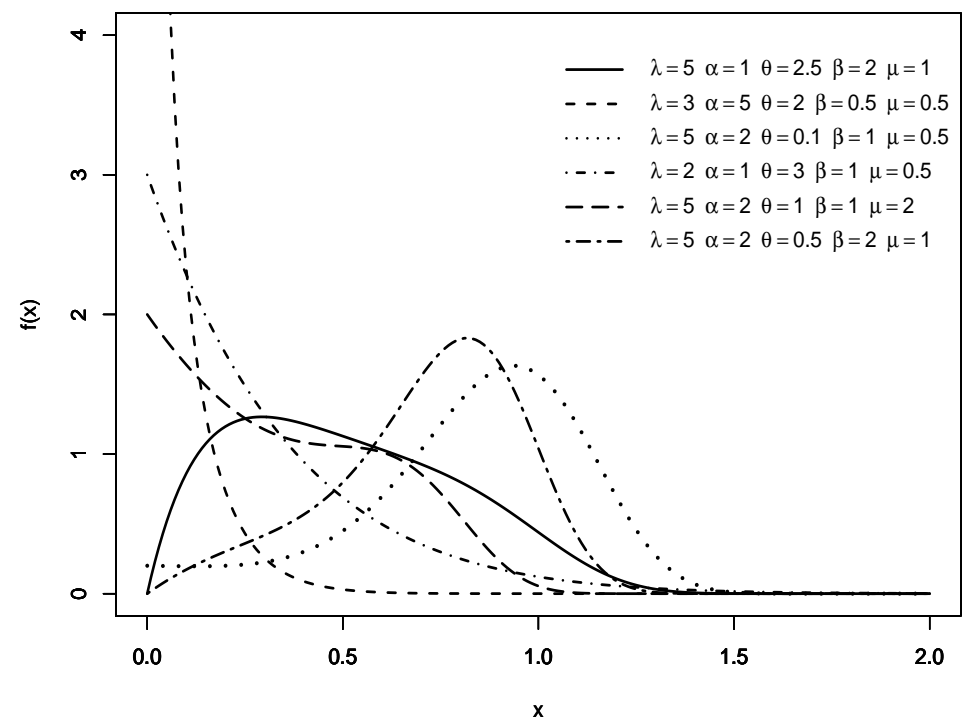

Figure 1: Probability density function of exponentiated generalized modified Weibull (EGMW) distribution.

\section{Exponentiated Generalized Modified Weibull Distribution}

In this section, we introduce the five-parameter exponentiated generalized modified Weibull (EGMW) distribution. Using (1.6) to (1.4), the cdf of the $\operatorname{EGMW}(\alpha, \theta, \mu, \lambda, \beta)$ distribution is

$$
\begin{aligned}
F(x) & =\left[1-\{1-G(x)\}^{\alpha}\right]^{\beta} \\
& =\left[1-e^{-\alpha\left(\theta x+\mu x^{\lambda}\right)}\right]^{\beta},
\end{aligned}
$$

where $\lambda>0, \theta, \mu>0$, such that $\theta+\mu>0$ and $\beta>0$.

The pdf of the new distribution is

$$
\begin{aligned}
f(x) & =\alpha \beta g(x)[1-G(x)]^{\alpha-1}\left[1-\{1-G(x)\}^{\alpha}\right]^{\beta-1} \\
& =\alpha \beta\left(\theta+\mu \lambda x^{\lambda-1}\right) e^{-\alpha\left(\theta x+\mu x^{\lambda}\right)}\left[1-e^{-\alpha\left(\theta x+\mu x^{\lambda}\right)}\right]^{\beta-1} .
\end{aligned}
$$

Figures 1 and 2, respectively, display the possible shape of the pdf and cdf of EGMW distribution for selected values of the parameters.

The reliability function and hazard (failure) rate function of the EGMW distribution are given by

$$
R(x)=1-F(x)=1-\left[1-e^{-\alpha\left(\theta x+\mu x^{\lambda}\right)}\right]^{\beta},
$$

and

$$
\begin{aligned}
h(x) & =\frac{f(x)}{1-F(x)} \\
& =\frac{\alpha \beta\left[\theta+\mu \lambda x^{\lambda-1}\right]}{\left[e^{\alpha\left(\theta x+\mu x^{\lambda}\right)}-1\right]\left[\left\{1-e^{-\alpha\left(\theta x+\mu x^{\lambda}\right)}\right\}^{-\beta}-1\right]}
\end{aligned}
$$




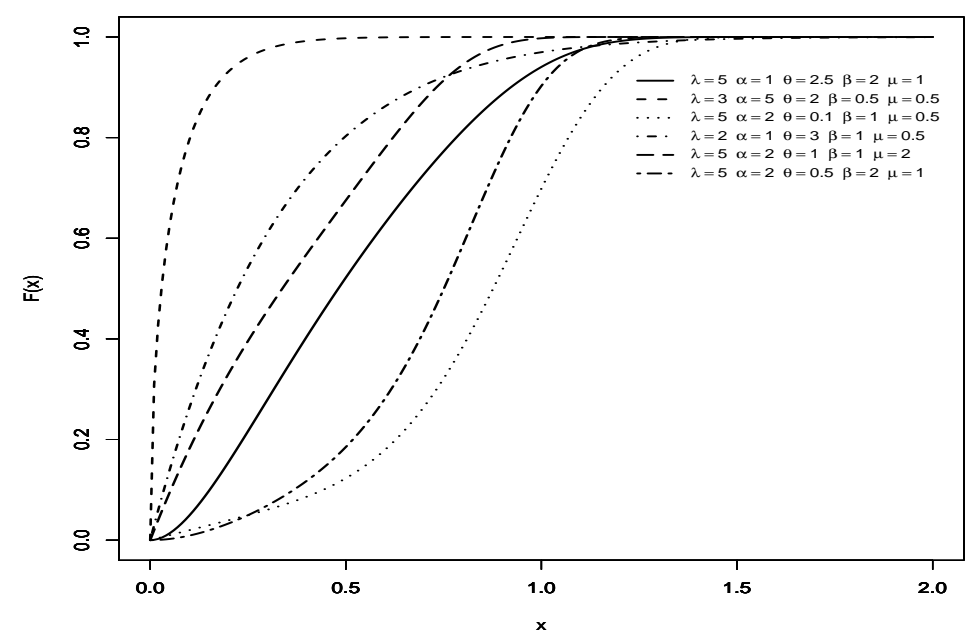

Figure 2: Cumulative distribution function of exponentiated generalized modified Weibull (EGMW) distribution.

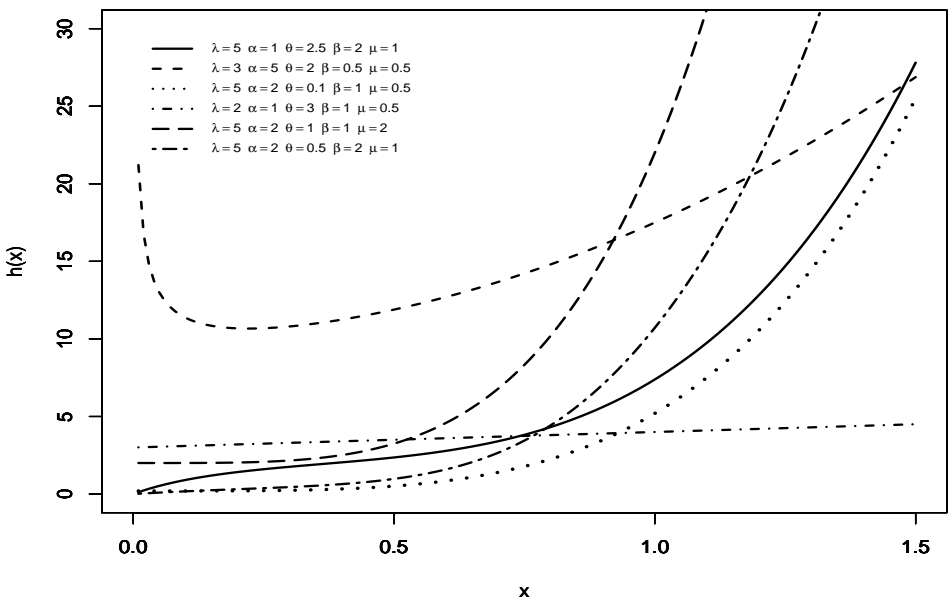

Figure 3: Hazard rate function of exponentiated generalized modified Weibull (EGMW) distribution.

respectively. Figure 3 illustrates the graphical behavior of the hazard rate function of EGMW distribution for selected values of the parameters.

Reversed hazard rate (RHR) function is an important reliability function applicable to various fields. Applications can be found in portfolio selection problems in finance, analysis of left-censored data, problems in actuarial science and forensic science involving estimation of exact time of occurrence of a particular event. For a random variable $X$ with pdf and cdf $f(x)$ and $F(x)$ respectively the RHR is given by

$$
r(x)=\frac{f(x)}{F(x)}
$$




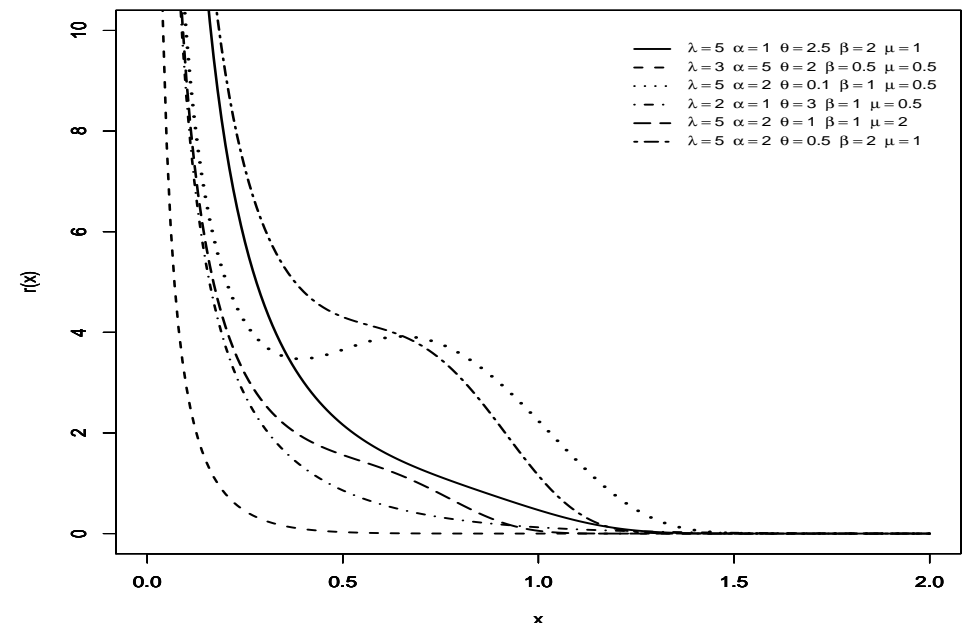

Figure 4: Reverse hazard rate of exponentiated generalized modified Weibull (EGMW) distribution.

Using (2.2) and (2.1) the reversed hazard rate function for a EGMW distribution is given by

$$
\begin{aligned}
r(x) & =\frac{\alpha \beta\left(\theta+\mu \lambda x^{\lambda-1}\right) e^{-\alpha\left(\theta x+\mu x^{\lambda}\right)}}{1-e^{-\alpha\left(\theta x+\mu x^{\lambda}\right)}} \\
& =\frac{\alpha \beta\left(\theta+\mu \lambda x^{\lambda-1}\right)}{e^{\alpha\left(\theta x+\mu x^{\lambda}\right)}-1} .
\end{aligned}
$$

Figure 4 illustrates the graphical behavior of the reversed hazard rate function of EGMW distribution for selected values of the parameters.

\subsection{Special distributions}

In this subsection we present distributions of special cases of the EGMW distribution for selected parameter values. The following are special cases:

- Exponentiated modified Weibull distribution: For $\alpha=1$, the EGMW distribution reduces to

$$
F(x)=\left[1-e^{-\left(\theta x+\mu x^{\lambda}\right)}\right]^{\beta},
$$

which is the cumulative distribution function of the EMW distribution introduced by Elbatal (2011).

- Generalized modified Weibull distribution: For $\beta=1$, the EGMW distribution reduces to

$$
F(x)=\left[1-e^{-\alpha\left(\theta x+\mu x^{\lambda}\right)}\right],
$$

which is the cumulative distribution function of the GMW distribution.

- Modified Weibull distribution: For $\alpha=\beta=1$, the EGMW distribution reduces to

$$
F(x)=1-e^{-\left(\theta x+\mu x^{\lambda}\right)},
$$


which is the cumulative distribution function of the MW distribution introduced by Mazen and Ammar (2009).

- Exponentiated Weibull distribution: For $\alpha=1$ and $\theta=0$, the EGMW distribution reduces to

$$
F(x)=\left[1-e^{-\mu x^{\lambda}}\right]^{\beta},
$$

which is the cumulative distribution function of the EW distribution introduced by Mudholkar and Srivastava (1993).

- Weibull distribution: For $\alpha=\beta=1$ and $\theta=0$, the EGMW distribution reduces to

$$
F(x)=1-e^{-\mu x^{2}},
$$

which is the cumulative distribution function of the two parameters Weibull distribution.

- Exponentiated exponential distribution: For $\alpha=\lambda=1$, and $\theta=0$, the EGMW distribution reduces to

$$
F(x)=\left[1-e^{-\mu x}\right]^{\beta},
$$

which is the cumulative distribution function of the exponentiated exponential distribution introduced by Gupta and Kundu (2001).

- Exponential distribution: For $\alpha=\lambda=\beta=1$, and $\theta=0$, the EGMW distribution reduces to

$$
F(x)=1-e^{-\mu x},
$$

which is the cumulative distribution function of the exponential distribution.

- Generalized linear failure rate distribution: For $\alpha=1$ and $\lambda=2$, the EGMW distribution reduces to

$$
F(x)=\left[1-e^{-\left(\theta x+\mu x^{2}\right)}\right]^{\beta},
$$

which is the cumulative distribution function of the generalized linear failure rate distribution introduced by Sarhan and Kundu (2009).

- Linear failure rate distribution: For $\alpha=\beta=1$ and $\lambda=2$, the EGMW distribution reduces to

$$
F(x)=1-e^{-\left(\theta x+\mu x^{2}\right)},
$$

which is the cumulative distribution function of the linear failure rate distribution.

- Generalized Rayleigh distribution: For $\alpha=1, \theta=0$ and $\lambda=2$, the EGMW distribution reduces to

$$
F(x)=\left[1-e^{-\mu x^{2}}\right]^{\beta},
$$

which is the cumulative distribution function of Burr Type $\mathrm{X}$ distribution, also known as the generalized Rayleigh distribution introduced by Surles and Padgett (2005).

- Rayleigh distribution: For $\alpha=\beta=1, \theta=0$ and $\lambda=2$, the EGMW distribution reduces to

$$
F(x)=1-e^{-\mu x^{2}},
$$

which is the cumulative distribution function of Rayleigh distribution. 


\subsection{Series representation}

In this subsection we present series representations of cdf and pdf of EGMW which will be useful to study the mathematical properties of the EGMW distribution. From the generalized binomial theorem we know that for $|z| \leq 1$,

$$
(1-z)^{b-1}=\sum_{j=0}^{\infty}(-1)^{j}\left(\begin{array}{c}
b-1 \\
j
\end{array}\right) z^{j}=\sum_{j=0}^{\infty} \frac{(-1)^{j} \Gamma(b)}{j ! \Gamma(b-j)} z^{j} .
$$

Also we know that

$$
e^{-x}=\sum_{k=0}^{\infty}(-1)^{k} \frac{x^{k}}{k !} .
$$

Applying (2.4) and (2.5) to (2.1) and (2.2) we have

$$
\begin{aligned}
F(x) & =\sum_{j=0}^{\infty}(-1)^{j}\left(\begin{array}{c}
\beta \\
j
\end{array}\right) e^{-\alpha j\left(\theta x+\mu x^{\lambda}\right)} \\
& =\sum_{k=0}^{\infty} \sum_{j=0}^{\infty}(-1)^{j+k}\left(\begin{array}{c}
\beta \\
j
\end{array}\right) \frac{(\alpha j)^{k}}{k !}\left(\theta x+\mu x^{\lambda}\right)^{k} .
\end{aligned}
$$

and

$$
\begin{aligned}
f(x) & =\alpha \beta \sum_{j=0}^{\infty} \frac{(-1)^{j} \Gamma(\beta)}{j ! \Gamma(\beta-j)}\left(\theta+\mu \lambda x^{\lambda-1}\right) e^{-\alpha(j+1)\left(\theta x+\mu x^{\lambda}\right)} \\
& =\alpha \beta \sum_{k=0}^{\infty} \sum_{j=0}^{\infty} \frac{(-1)^{j+k} \Gamma(\beta)}{\Gamma(\beta-j)} \frac{(\alpha j+\alpha)^{k}}{j ! k !}\left(\theta+\mu \lambda x^{\lambda-1}\right)\left(\theta x+\mu x^{\lambda}\right)^{k} .
\end{aligned}
$$

\section{Statistical Properties}

This section examines statistical properties of EGMW distribution such as, quantile function, moments and moment generating function.

\subsection{Quantile function}

The quantile $x_{q}$ of the EGMW $(\alpha, \theta, \mu, \lambda, \beta)$ distribution is the solution for:

$$
\alpha\left(\theta x_{q}+\mu x_{q}^{\lambda}\right)+\ln \left(1-q^{\frac{1}{\beta}}\right)=0 .
$$

The above equation has no closed form analytical solution in $x_{q}$, but we can use computational facilities available in programming software like R, Python, MATLAB, Maple, Mathematica to find numerical solution for the quantiles.

\subsection{Moments}

In this subsection we discuss the $r^{\text {th }}$ moment for EGMW distribution. Moments are necessary and important for statistical analysis to study the important features and characteristics of a distribution (e.g., tendency, dispersion, skewness and kurtosis). Simple calculation yields the following. 
If $X$ has $\operatorname{EGMW}(\alpha, \beta, \theta, \mu, \lambda)$ distribution, then its $r^{\text {th }}$ order moment is given by

$$
E\left(X^{r}\right)=\alpha \beta \sum_{i=0}^{\infty} \sum_{j=0}^{\infty} \frac{(-1)^{i+j} \Gamma(\beta)(\alpha(j+1) \mu)^{i}}{i ! j ! \Gamma(\beta-j)}\left\{\frac{\theta \Gamma(r+\lambda i+1)}{(\alpha \theta(j+1))^{r+\lambda i+1}}+\frac{\lambda \mu \Gamma(r+\lambda(i+1))}{(\alpha \theta(j+1))^{r+\lambda(i+1)}}\right\} .
$$

Similarly, the moment generating function of EGMW distribution is given by

$$
M_{X}(t)=\alpha \beta \sum_{i=0}^{\infty} \sum_{j=0}^{\infty} \frac{(-1)^{i+j} \Gamma(\beta)(\alpha(j+1) \mu)^{i}}{i ! j ! \Gamma(\beta-j)}\left\{\frac{\theta \Gamma(\lambda i+1)}{(\alpha \theta(j+1)-t)^{\lambda i+1}}+\frac{\lambda \mu \Gamma(\lambda(i+1))}{(\alpha \theta(j+1)-t)^{\lambda(i+1)}}\right\} .
$$

\section{Order Statistics}

Let $X_{1}, X_{2}, \ldots, X_{n}$ be a simple random sample from EGMW distribution with cdf and pdf given by (2.1) and (2.2), respectively. Let $X_{(1: n)} \leq X_{(2: n)} \leq \cdots \leq X_{(n: n)}$ denote the order statistics obtained from this sample. We now give the probability density function of $X_{i: n}$, say $f_{i: n}(x)$ of $X_{i: n}$ for $i=1,2, \ldots, n$. The probability density function of $X_{i: n}$ is given by

$$
f_{i: n}(x)=\frac{1}{B(i, n-i+1)}[F(x, \Theta)]^{i-1}[1-F(x, \Theta)]^{n-i} f(x, \Theta),
$$

where $\Theta=(\alpha, \beta, \theta, \mu, \lambda)$ and $F(x, \Theta)$ and $f(x, \Theta)$ are the cdf and pdf of the EGMW distribution respectively, and $B(\cdot, \cdot)$ is the beta function. We have

$$
f_{i: n}(x)=\frac{\alpha \beta}{B(i, n-i+1)} g(x)\{1-G(x)\}^{\alpha-1}\left[1-\{1-G(x)\}^{\alpha}\right]^{\beta i-1}\left\{1-\left[1-\{1-G(x)\}^{\alpha}\right]^{\beta}\right\}^{n-i} .
$$

Using the binomial series expansion, $f_{i: n}(x)$ can be expressed as

$$
f_{i: n}(x)=\sum_{k=0}^{n-i}(-1)^{k}\left(\begin{array}{c}
n-i \\
k
\end{array}\right) \frac{\alpha \beta}{B(i, n-i+1)} g(x)\{1-G(x)\}^{\alpha-1}\left[1-\{1-G(x)\}^{\alpha}\right]^{\beta(i+k)-1} .
$$

Again applying (2.4) to the last term, we get

$$
\begin{aligned}
f_{i: n}(x) & =\sum_{k=0}^{n-i} \sum_{m=0}^{\infty}(-1)^{k+m}\left(\begin{array}{c}
n-i \\
k
\end{array}\right)\left(\begin{array}{c}
\beta(i+k)-1 \\
m
\end{array}\right) \frac{\alpha \beta}{B(i, n-i+1)} g(x)\{1-G(x)\}^{\alpha(m+1)-1} \\
& =\sum_{k=0}^{n-i} \sum_{s=0}^{\infty} \sum_{m=0}^{\infty}(-1)^{k+s+m}\left(\begin{array}{c}
n-i \\
k
\end{array}\right)\left(\begin{array}{c}
\beta(i+k)-1 \\
m
\end{array}\right)\left(\begin{array}{c}
\alpha(m+1)-1 \\
s
\end{array}\right) \frac{\alpha \beta}{B(i, n-i+1)} g(x) G(x)^{s} \\
& =w_{k s m} \frac{\alpha \beta}{B(i, n-i+1)} g(x) G(x)^{s},
\end{aligned}
$$

where

$$
w_{k s m}=\sum_{k=0}^{n-i} \sum_{s=0}^{\infty} \sum_{m=0}^{\infty}(-1)^{k+s+m}\left(\begin{array}{c}
n-i \\
k
\end{array}\right)\left(\begin{array}{c}
\beta(i+k)-1 \\
m
\end{array}\right)\left(\begin{array}{c}
\alpha(m+1)-1 \\
s
\end{array}\right) .
$$

$G(x)$ and $g(x)$ in (4.4) are the cdf and the pdf of the MW distribution given in (1.6) and (1.7) respectively. The pdf of the order statistics are observed to be equal to the density of MW distribution multiplied by an infinite weighted power series of cdfs for the MW distribution. 


\subsection{Residual life and reversed failure rate function}

Given that a component survives up to time $t \geq 0$, the residual life is the period beyond $t$ until the time of failure and defined by the conditional random variable $X-t$ given that $X>t$ and is denoted as $X-t \mid X>t$. In reliability analysis, it is well known that the mean residual life function $E(X-t \mid X>t)$ determines the distribution uniquely but Gupta and Gupta (1983) presented a method which can be used to determine the distribution if the ratio two consecutive moments is known. Therefore, we obtain the $r^{\text {th }}$-order moment of the residual life using the general formula

$$
\mu_{r}(t)=E\left[(X-t)^{r} \mid X>t\right]=\frac{1}{\bar{F}(t)} \int_{t}^{\infty}(x-t)^{r} f(x, \Theta) d x, \quad r \geq 1 .
$$

Applying the binomial expansion of $(x-t)^{r}$ and substituting $f(x, \Theta)$ given by (2.7) into the above formula we get

$$
\mu_{r}(t)=\frac{\alpha \beta}{\bar{F}(t)} \sum_{i=0}^{r} \sum_{j=0}^{\infty} \frac{(-1)^{i+j} \Gamma(\beta) t^{i}}{j ! \Gamma(\beta-j)}\left(\begin{array}{l}
r \\
i
\end{array}\right) \int_{t}^{\infty} x^{r-i}\left(\theta+\mu \lambda x^{\lambda-1}\right) e^{-\alpha(j+1)\left(\theta x+\mu x^{\lambda}\right)} d x
$$

using Equation (3.1) we get

$$
\begin{aligned}
& \mu_{r}(t)=\frac{\alpha \beta}{\bar{F}(t)} \sum_{i=0}^{r} \sum_{j=0}^{\infty} \sum_{k=0}^{\infty} \frac{(-1)^{i+j+k} \Gamma(\beta)(\alpha(j+1) \mu)^{k} t^{i}}{j ! k ! \Gamma(\beta-j)}\left(\begin{array}{l}
r \\
i
\end{array}\right) \int_{t}^{\infty} x^{r+\lambda k-i}\left(\theta+\mu \lambda x^{\lambda-1}\right) e^{-\alpha(j+1) \theta x} d x \\
& =\frac{\alpha \beta}{\bar{F}(t)} \sum_{i=0}^{r} \sum_{j=0}^{\infty} \sum_{k=0}^{\infty} \frac{(-1)^{i+j+k} \Gamma(\beta)(\alpha(j+1) \mu)^{k} t^{i}}{j ! k ! \Gamma(\beta-j)}\left(\begin{array}{l}
r \\
i
\end{array}\right) \\
& \times\left[\frac{\theta \Gamma(r-i+\lambda k+1, \alpha(j+1) \theta t)}{(\alpha(j+1) \theta t)^{r-i+\lambda k+1}}+\mu \lambda \frac{\Gamma(r-i+\lambda(k+1), \alpha(j+1) \theta t)}{(\alpha(j+1) \theta t)^{r-i+\lambda(k+1)}}\right],
\end{aligned}
$$

where $\Gamma(s, t)=\int_{t}^{\infty} x^{s-1} e^{-x} d x$ is the upper incomplete gamma function. Therefore, the mean residual life of the EGMW distribution is given by

$$
\begin{aligned}
\mu_{1}(t)= & \frac{\alpha \beta}{\bar{F}(t)} \sum_{j=0}^{\infty} \sum_{k=0}^{\infty} \frac{(-1)^{j+k} \Gamma(\beta)(\alpha(j+1) \mu)^{k}}{j ! k ! \Gamma(\beta-j)}\left[\frac{\theta \Gamma(\lambda k+2, \alpha(j+1) \theta t)}{(\alpha(j+1) \theta t)^{\lambda k+2}}\right. \\
& \left.+\mu \lambda \frac{\Gamma(\lambda(k+1)+1, \alpha(j+1) \theta t)}{(\alpha(j+1) \theta t)^{\lambda(k+1)+1}}\right]-t .
\end{aligned}
$$

However, reversed residual life can be defined as the conditional random variable $t-X$ given that $X \leq t$ and is denoted by $t-X \mid X \leq t$. This denotes the time elapsed from the failure of a component given that its life is less than or equal to $t$. This random variable may also be called the inactivity time (or time since failure); for more details readers are refer to Nanda et al. (2003). In reliability, the mean reversed residual life and ratio of two consecutive moments of reversed residual life characterize the distribution uniquely. The reverse failure(or reversed hazard) rate function is given in (2.3). The $r^{\text {th }}$-order moment of the reversed residual life can be obtained by the well known formula

$$
m_{r}(t)=E\left[(t-X)^{r} \mid X \leq t\right]=\frac{1}{\bar{F}(t)} \int_{0}^{t}(t-x)^{r} f(x, \Theta) d x, \quad r \geq 1 .
$$


Applying the binomial expansion of $(t-x)^{r}$ and substituting $f(x, \Theta)$ given by (2.7) into the above formula gives

$$
\begin{aligned}
& m_{r}(t)=\frac{\alpha \beta}{\bar{F}(t)} \sum_{i=0}^{r} \sum_{j=0}^{\infty} \sum_{k=0}^{\infty} \frac{(-1)^{i+j+k} \Gamma(\beta)(\alpha(j+1) \mu)^{k} t^{i}}{j ! k ! \Gamma(\beta-j)}\left(\begin{array}{l}
r \\
i
\end{array}\right) \int_{0}^{t} x^{r+\lambda k-i}\left(\theta+\mu \lambda x^{\lambda-1}\right) e^{-\alpha(j+1) \theta x} d x \\
& =\frac{\alpha \beta}{\bar{F}(t)} \sum_{i=0}^{r} \sum_{j=0}^{\infty} \sum_{k=0}^{\infty} \frac{(-1)^{i+j+k} \Gamma(\beta)(\alpha(j+1) \mu)^{k} t^{i}}{j ! k ! \Gamma(\beta-j)}\left(\begin{array}{l}
r \\
i
\end{array}\right) \\
& \times\left[\frac{\theta \Gamma(r-i+\lambda k+1, \alpha(j+1) \theta t)}{(\alpha(j+1) \theta t)^{r-i+\lambda k+1}}+\mu \lambda \frac{\Gamma(r-i+\lambda(k+1), \alpha(j+1) \theta t)}{(\alpha(j+1) \theta t)^{r-i+\lambda(k+1)}}\right],
\end{aligned}
$$

where $\Gamma(s, t)=\int_{0}^{t} x^{s-1} e^{-x} d x$ is the lower incomplete gamma function. In particular, the mean of the reversed residual life of the EGMW distribution is given by

$$
\begin{aligned}
m_{1}(t)= & t-\frac{\alpha \beta}{\bar{F}(t)} \sum_{j=0}^{\infty} \sum_{k=0}^{\infty} \frac{(-1)^{j+k} \Gamma(\beta)(\alpha(j+1) \mu)^{k}}{j ! k ! \Gamma(\beta-j)}\left[\frac{\theta \Gamma(\lambda k+2, \alpha(j+1) \theta t)}{(\alpha(j+1) \theta t)^{\lambda k+2}}\right. \\
& \left.+\mu \lambda \frac{\Gamma(\lambda(k+1)+1, \alpha(j+1) \theta t)}{(\alpha(j+1) \theta t)^{\lambda(k+1)+1}}\right] .
\end{aligned}
$$

\section{Estimation and Inference}

In this section, we determine the maximum likelihood estimates (MLEs) of the parameters of the EGMW distribution from complete samples only. Let $X_{1}, X_{2}, \ldots, X_{n}$ be a random sample of size $n$ from EGMW $(\Theta, x)$, where $\Theta=(\alpha, \beta, \theta, \mu, \lambda)$. The $\log$-likelihood function $\log (L)=\ell$ for the vector of parameters $\Theta=(\alpha, \beta, \theta, \mu, \lambda)$ can be written as

$$
\begin{aligned}
\ell= & n \log \alpha+n \log \beta+\sum_{i=1}^{n} \log \left(\theta+\mu \lambda x_{i}^{\lambda-1}\right)-\alpha \theta \sum_{i=1}^{n} x_{i} \\
& -\alpha \mu \sum_{i=1}^{n} x_{i}^{\lambda}+(\beta-1) \sum_{i=1}^{n} \log \left[1-e^{-\alpha\left(\theta x_{i}+\mu x_{i}^{\lambda}\right)}\right] .
\end{aligned}
$$

The log-likelihood can be maximized either directly or by solving the nonlinear likelihood equations obtained by differentiating (5.1). The components of the score vector are given by

$$
\begin{aligned}
& \frac{\partial \ell}{\partial \alpha}=\frac{n}{\alpha}-\theta \sum_{i=1}^{n} x_{i}-\mu \sum_{i=1}^{n} x_{i}^{\lambda}+(\beta-1) \sum_{i=1}^{n} \frac{\left(\theta x_{i}+\mu x_{i}^{\lambda}\right) e^{-\alpha\left(\theta x_{i}+\mu x_{i}^{\lambda}\right)}}{\left[1-e^{\left.-\alpha\left(\theta x_{i}+\mu x_{i}^{\lambda}\right)\right]},\right.} \\
& \frac{\partial \ell}{\partial \theta}=\sum_{i=1}^{n} \frac{1}{\left(\theta+\mu \lambda x_{i}^{\lambda-1}\right)}-\alpha \sum_{i=1}^{n} x_{i}+\alpha(\beta-1) \sum_{i=1}^{n} \frac{x_{i} e^{-\alpha\left(\theta x_{i}+\mu x_{i}^{\lambda}\right)}}{\left[1-e^{\left.-\alpha\left(\theta x_{i}+\mu x_{i}^{\lambda}\right)\right]}\right.}, \\
& \frac{\partial \ell}{\partial \mu}=\lambda \sum_{i=1}^{n} \frac{x_{i}^{\lambda-1}}{\left(\theta+\mu \lambda x_{i}^{\lambda-1}\right)}-\alpha \sum_{i=1}^{n} x_{i}^{\lambda}+\alpha(\beta-1) \sum_{i=1}^{n} \frac{x_{i}^{\lambda} e^{-\alpha\left(\theta x_{i}+\mu x_{i}^{\lambda}\right)}}{\left[1-e^{-\alpha\left(\theta x_{i}+\mu x_{i}^{\lambda}\right)}\right]}
\end{aligned}
$$


Table 1: Empirical means and the RMSEs of the EGMW distribution for $\alpha=1.5, \beta=2, \theta=0.5, \mu=1.5 \&$ $\lambda=3.0$

\begin{tabular}{|c|c|c|c|c|c|}
\hline$n$ & $\hat{\alpha}$ & $\hat{\beta}$ & $\hat{\theta}$ & $\hat{\mu}$ & $\hat{\lambda}$ \\
\hline \multirow{2}{*}{100} & 1.402 & 3.163 & 1.151 & 1.374 & 4.002 \\
\hline & $(0.244)$ & (2.053) & (1.214) & $(0.351)$ & (1.889) \\
\hline \multirow{2}{*}{200} & 1.423 & 2.660 & 0.915 & 1.368 & 3.584 \\
\hline & $(0.167)$ & (1.532) & $(0.927)$ & $(0.272)$ & (1.081) \\
\hline \multirow{2}{*}{300} & 1.429 & 2.528 & 0.859 & 1.369 & 3.428 \\
\hline & $(0.144)$ & (1.111) & $(0.722)$ & $(0.243)$ & $(0.767)$ \\
\hline \multirow{2}{*}{500} & 1.464 & 2.362 & 0.724 & 1.408 & 3.226 \\
\hline & $(0.097)$ & $(0.888)$ & $(0.504)$ & $(0.192)$ & $(0.406)$ \\
\hline \multirow{2}{*}{1000} & 1.478 & 2.049 & 0.554 & 1.467 & 3.132 \\
\hline & $(0.054)$ & $(0.565)$ & $(0.329)$ & (0.116) & $(0.264)$ \\
\hline
\end{tabular}

RMSE = root mean square error; EGMW = exponentiated generalized modified Weibull.

$$
\begin{aligned}
& \frac{\partial \ell}{\partial \lambda}=\mu \sum_{i=1}^{n} \frac{x_{i}^{\lambda-1}\left(\lambda \log x_{i}+1\right)}{\left(\theta+\mu \lambda x_{i}^{\lambda-1}\right)}-\alpha \mu \sum_{i=1}^{n} x_{i}^{\lambda} \log x_{i}+\alpha \mu(\beta-1) \sum_{i=1}^{n} \frac{e^{-\alpha\left(\theta x_{i}+\mu x_{i}^{\lambda}\right)} x_{i}^{\lambda} \log x_{i}}{\left[1-e^{-\alpha\left(\theta x_{i}+\mu x_{i}^{\lambda}\right)}\right]} \\
& \frac{\partial \ell}{\partial \beta}=\frac{n}{\beta}+\sum_{i=1}^{n} \log \left[1-e^{-\alpha\left(\theta x_{i}+\mu x_{i}^{\lambda}\right)}\right] .
\end{aligned}
$$

The maximum likelihood estimators $\hat{\alpha}, \hat{\beta}, \hat{\theta}, \hat{\mu}$ and $\hat{\lambda}$ of $\alpha, \beta, \theta, \mu$ and $\lambda$ are obtained by setting the score vector (5.2)-(5.6) to zero and solving the system of nonlinear equations. It is usually more convenient to use nonlinear optimization algorithms like the quasi-Newton algorithm to numerically maximize the log-likelihood function given in (5.1). Also, note that all the second order derivatives exist. We can compute the maximum values of the unrestricted and restricted log-likelihood functions to obtain likelihood ratio (LR) statistics to test the sub-model of the new distribution.

\section{Simulation Study}

In this section, we provide the simulation results to assess the performance of the proposed maximum likelihood estimation procedure. An ideal technique for simulating from (2.2) is the inversion method. One would simulate $X$ by solving

$$
\alpha\left(\theta x+\mu x^{\lambda}\right)+\ln \left(1-u^{\frac{1}{\beta}}\right)=0,
$$

where $u \sim U(0,1)$ is a uniformly distributed. For different combination of $\alpha, \beta, \theta, \mu$ and $\lambda$ samples of sizes $n=100,200,300,500$ and 1000 are generated from the EGMW distribution. We repeated the simulation $k=100$ times and calculated the mean and the root mean square errors (RMSEs). The limited- memory quasi-Newton with bound-constrained optimization (L-BFGS-B) method has been used to maximize the total log-likelihood function. The required computations use a script Adequacy Model of the R-package written by Marinho et al. (2015). Table 1 provides the empirical results of the simulation. The estimates prove to be quite stable and are close to the true value of the parameters for the sample sizes; in addition, the RMSEs, provided in the parentheses, decreases as the sample size increases.

\section{Applications}

In this section, we provide a data analysis in order to assess the goodness-of-fit of the model. In particular, we would like to present a data where the proposed EGMW exhibits an improvement 
Table 2: Descriptive statistics of the failure time

\begin{tabular}{cccccccc}
\hline \hline$n$ & Mean & Median & Variance & Minimum & Maximum & Skewness & Kurtosis \\
\hline 50 & 3.34296 & 1.414 & 17.48477 & 0.036 & 15.08 & 1.416739 & 4.084622 \\
\hline \hline
\end{tabular}

Table 3: Estimated parameters for the failure times

\begin{tabular}{cccccc}
\hline \hline Model & $\hat{\beta}$ & $\hat{\alpha}$ & $\hat{\theta}$ & $\hat{\mu}$ & $\hat{\lambda}$ \\
\hline \multirow{2}{*}{ EGMWD } & 89.060 & 34.428 & 0.004 & 0.133 & 0.093 \\
& $(780.393)$ & $(32.861)$ & $(0.003)$ & $(0.170)$ & $(0.184)$ \\
\hline \multirow{2}{*}{ GMWD } & - & 0.666 & 0.066 & 0.740 & 0.620 \\
& - & $(31.008)$ & $(3.076)$ & $(34.464)$ & $(0.155)$ \\
\hline \hline
\end{tabular}

EGMWD = exponentiated generalized modified Weibull distribution; GMWD = generalized modified Weibull distribution.

over the generalized modified Weibull (GMW) distribution. The data set given below are taken from Murthy et al. (2004) and represent the failure times of 50 components (per 1000h). The observations are:

0.036, 0.058, 0.061, 0.074, 0.078, 0.086, 0.102, 0.103, 0.114, 0.116, 0.148, 0.183, 0.192, 0.254, 0.262, $0.379,0.381,0.538,0.570,0.574,0.590,0.618,0.645,0.961,1.228,1.600,2.006,2.054,2.804,3.058$, $3.076,3.147,3.625,3.704,3.931,4.073,4.393,4.534,4.893,6.274,6.816,7.896,7.904,8.022,9.337$, $10.940,11.020,13.880,14.730,15.080$.

Table 2 provides the descriptive summary of the data. We compute the maximum likelihood estimates (MLEs) for both EGMW and GMW distributions using L-BFGS-B as described above. The goodness.fit command allows the computation of standard errors of the model parameters based on the observed fisher information matrix. The square root of the diagonal elements of the fisher information matrix are the standard errors. Table 3 lists the estimates of the parameters and their standard errors (in parentheses) for both the EGMW distribution and GMW distribution.

Model selection uses: Akaike information criterion(AIC), Bayesian information criterion(BIC), consistent Akaike information criterion(CAIC) and Hannan-Quinn information criterion(HQIC). These measures are defined as

$$
\begin{aligned}
\mathrm{AIC} & =-2 \ell(\hat{\Theta})+2 q, \\
\mathrm{BIC} & =-2 \ell(\hat{\Theta})+q \log (n), \\
\mathrm{HQIC} & =-2 \ell(\hat{\Theta})+2 q \log (\log (n)), \\
\mathrm{CAIC} & =-2 \ell(\hat{\Theta})+\frac{2 q n}{n-q-1},
\end{aligned}
$$

where $\ell(\hat{\Theta})$ denotes the log-likelihood function evaluated at the maximum likelihood estimates, $q$ is the number of parameters, and $n$ is the sample size. Here $\Theta$ denote the parameters. In addition, we calculate Anderson-Darling $\left(A^{*}\right)$ and Cramèr-von Mises $\left(W^{*}\right)$ statistics. Note that the smaller the values of goodness-of-fit measures better the fit of the data is. Table 4 lists the numerical values of $-\ell$, AIC, CAIC, HQIC, BIC, $A^{*}$ and $W^{*}$.

The value of negative log-likelihood function evaluated at the maximum likelihood estimates $(-\ell(\hat{\Theta}))$ along with all other statistics for the EGMW distribution are smaller than for the sub-model GMW distribution. We employ the LR statistic to test the superiority of the EGMW distribution over the GMW distribution. To perform this test the maximized restricted and unrestricted log-likelihoods 
Table 4: The statistics $-\ell$, AIC ,CAIC, HQIC, BIC, $A^{*}$ and $W^{*}$ for failure time data

\begin{tabular}{cccccccc}
\hline \hline \multirow{2}{*}{ Model } & \multicolumn{7}{c}{ Goodness of fit criteria } \\
\cline { 2 - 8 } & $-\ell(\cdot)$ & AIC & CAIC & HQIC & BIC & $A^{*}$ & $W^{*}$ \\
\hline EGMWD & 99.696 & 209.391 & 210.755 & 213.032 & 218.951 & 0.724 & 0.115 \\
GMWD & 102.320 & 212.641 & 213.530 & 215.553 & 220.289 & 0.945 & 0.150 \\
\hline \hline
\end{tabular}

AIC = Akaike information criterion; CAIC = consistent Akaike information criteria; BIC = Bayesian information criterion; HQIC = Hannan-Quinn information criterion (HQIC); EGMWD = exponentiated generalized modified Weibull distribution; GMWD = generalized modified Weibull distribution .

Table 5: The Kolmogorov-Smirnov (KS) test for failure time data

\begin{tabular}{ccc}
\hline \hline Model & \multicolumn{2}{c}{ Goodness of fit criteria } \\
\cline { 2 - 3 } & KS Statistic & $p$-value \\
\hline EGMWD & 0.1038 & 0.6165 \\
GMWD & 0.1279 & 0.3564 \\
\hline \hline EGMWD = exponentiated generalized modified Weibull distribution; GMWD = generalized modified Weibull distribution
\end{tabular}

can be computed under the null and alternative hypothesis

$$
\begin{aligned}
& H_{0}: \beta=1 \text { (restricted, GMW model is true for the data set), } \\
& H_{a}: \beta \neq 1 \text { (unrestricted, EGMW model is true for the data set). }
\end{aligned}
$$

The LR test statistic for testing $H_{0}$ versus $H_{a}$ is

$$
\omega=2\left(\ell(\hat{\Theta}, x)-\ell\left(\hat{\Theta}_{0}, x\right)\right),
$$

where $\hat{\Theta}$ and $\hat{\Theta}_{0}$ are the MLEs under $H_{a}$ and $H_{0}$, respectively. The statistic $\omega$ is asymptotically distributed as $\chi_{v}^{2}$. Here $v$ is the difference in the numbers of parameter for restricted and unrestricted models. The computed value of the LR statistic under the hypothesis is $\omega=5.248$ and $v=1$. Note that $\chi_{0.05,1}^{2}=3.84$. Therefore, we reject the null hypothesis $\left(H_{0}\right)$ at a $5 \%$ level of significance and conclude that the EGMW model fits better for the subject data set.

Table 5 provides the Kolmogorov-Smirnov (KS) test statistic and corresponding $p$-values for both EGMW distribution and GMW distribution. The KS test statistic for EGMW distribution is lower than that for GMW distribution and it indicates that the EGMW model fits the data better than the GMW model.

Figure 5 provides the plots to compare the exact EGMW distribution, GMW distribution and empirical cdf along with the P-P plot for this data. These plots indicate that EGMW distribution fits the subject data better than GMW distribution.

\section{Concluding Remarks}

In the present study, we have introduced a new generalization of the modified Weibull distribution the so-called exponentiated generalized modified Weibull distribution. Standard distributions such as exponential, Rayleigh, Weibull and linear failure along with their exponentiated versions are embedded in the proposed distribution. Some mathematical properties along with estimation issues are also addressed. We have presented an example to illustrate the application of the subject distribution to model the reliability data. The proposed distribution appears to include several well-known distributions that can model data that arises from several different areas. We expect that this study can serve as a reference to help advance future research in the subject area. 

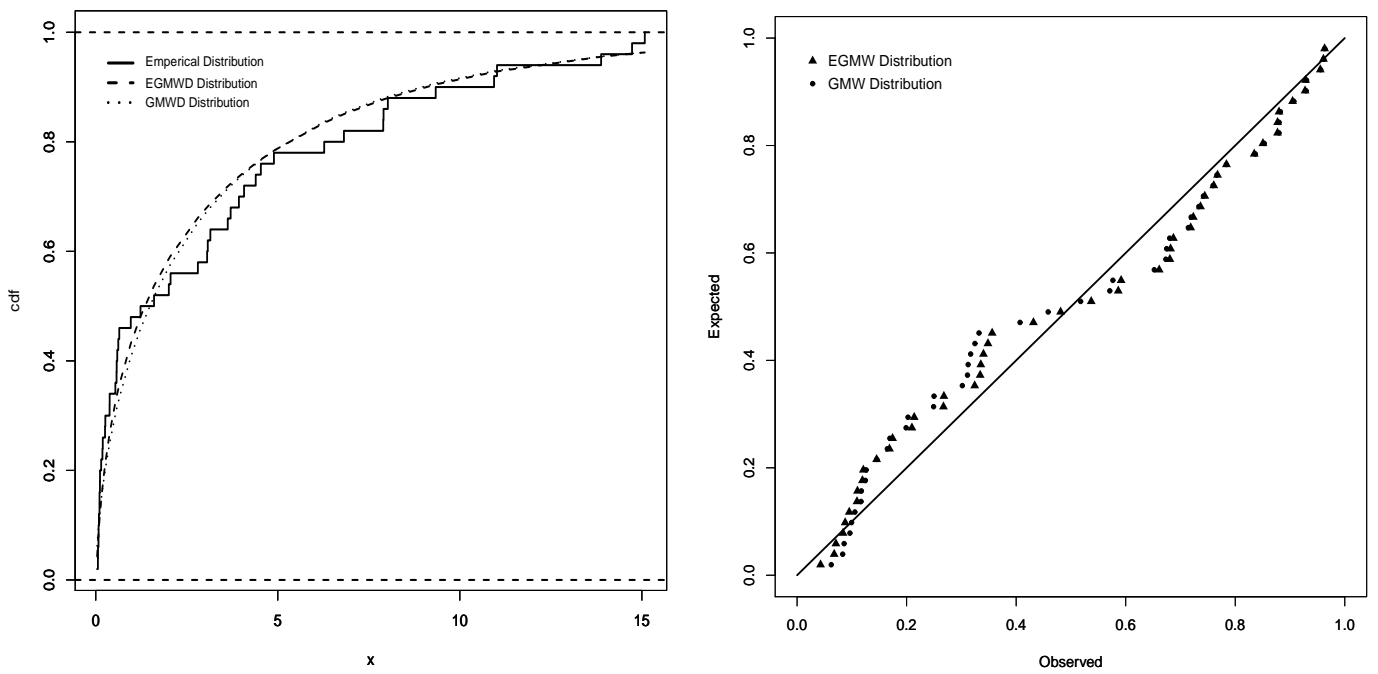

Figure 5: Fitted cumulative distribution function and P-P plot of exponentiated generalized modified Weibull $(E G M W)$ distribution and generalized modified Weibull (GMW) distribution.

\section{Acknowledgements}

The authors would like to thank the associate editor and three anonymous reviewers for carefully reading the manuscript and making valuable suggestions.

\section{References}

Carrasco, M., Ortega, E. M. and Cordeiro, G. M. (2008). A generalized modified Weibull distribution for lifetime modeling, Computational Statistics \& Data Analysis, 53, 450-462.

Cordeiro, G. M., Ortega, E. M. M. and da Cunha, D. C. (2013). The exponentiated generalized class of distributions, Journal of Data Science, 11, 1-27.

Elbatal, I. (2011). Exponentiated modified Weibull distribution, Economic Quality Control, 26, 189200.

Eugene, N., Lee, C. and Famoye, F. (2002). Beta-normal distribution and its applications, Communications in Statistics - Theory and Methods, 31, 497-512.

Famoye, F., Lee, C. and Olumolade, O. (2005). The beta-Weibull distribution, Journal of Statistical Theory and Applications, 4, 121-138.

Gupta, P. L. and Gupta, R. C. (1983). On the moments of residual life in reliability and some characterization results, Communications in Statistics - Theory and Methods, 12, 449-461.

Gupta, R. C., Gupta, P. L. and Gupta, R. D. (1998). Modeling failure time data by Lehman alternatives, Communications in Statistics - Theory and Methods, 27, 887-904.

Gupta, R. D. and Kundu, D. (2001). Exponentiated exponential family: An alternative to gamma and Weibull distributions, Biometrical Journal, 43, 117-130.

Hanook, S., Shahbaz, M. Q., Mohsin, M. and Golam Kibria, B. M. (2013). A note on beta InverseWeibull Distribution, Communications in Statistics - Theory and Methods, 42, 320-335.

Jones, M. C. (2009). Kumaraswamy's distribution: A beta-type distribution with some tractability advantages, Statistical Methodology, 6, 70-81.

Lai, C. D., Xie, M. and Murthy, D. N. P. (2003). A modified Weibull distribution, IEEE Transactions 
on Reliability, 52, 33-37.

Lehmann, E. L. (1953). The power of rank tests, Annals of Mathematical Statistics, 24, 23-43.

Marinho, P. R. D., Bourguignon, M. and Dias, C. R. B. (2015). AdequacyModel 1.0.8: Adequacy of probabilistic models and generation of pseudo-random numbers, Available from: http://cran.rproject.org/web/packages/AdequacyModel/AdequacyModel.pdf.

Mazen, Z. and Ammar, M. (2009). Parameters estimation of the modified Weibull distribution, Applied Mathematical Sciences, 3, 541-550.

Mudholkar, G. S. and Srivastava, D. K. (1993). Exponentiated Weibull family for analyzing bathtub failure-rate data, IEEE Transactions on Reliability, 42, 299-302.

Murthy, D. N. P., Xie, M. and Jiang, R. (2004). Weibull Models, John Wiley \& Sons Inc., Hoboken, NJ.

Nadarajah, S., Cordeiro, G. M. and Ortega, E. M. M. (2011). General results for the beta-modified Weibull distribution, Journal of Statistical Computation and Simulation, 81, 1211-1232.

Nadarajah, S. and Gupta, A. K. (2004). The beta Fr?chet distribution, Far East Journal of Theoretical Statistics, 14, 15-24.

Nadarajah, S. and Kotz, S. (2004). The beta Gumbel distribution, Mathematical Problems in Engineering, 2004, 323-332.

Nadarajah, S. and Kotz, S. (2006). The exponentiated type distributions, Acta Applicandae Mathematica, 92, 97-111.

Nanda, A. K., Singh, H., Misra, N. and Paul, P. (2003). Reliability properties of reversed residual lifetime, Communications in Statistics - Theory and Methods, 32, 2031-2042.

Sarhan, A. M. and Kundu, D. (2009). Generalized linear failure rate distribution, Communications in Statistics - Theory and Methods, 38, 642-660.

Sarhan, A. M. and Zaindin, M. (2009). Modified Weibull distribution, Applied Sciences, 11, 123-136.

Surles, J. G. and Padgett, W. J. (2005). Some properties of a scaled Burr type X distribution, Journal of Statistical Planning and Inference, 128, 271-280. 